\title{
Comparison of Accelerometry Cut Points for Physical Activity and Sedentary Behavior in Preschool Children: A Validation Study
}

\author{
Jane F. Hislop, Cathy Bulley, and Tom H. Mercer \\ Queen Margaret University \\ John J. Reilly \\ University of Strathclyde
}

\begin{abstract}
This study compared accelerometry cut points for sedentary behavior, light and moderate to vigorous intensity activity (MVPA) against a criterion measure, the Children's Activity Rating Scale (CARS), in preschool children. Actigraph accelerometry data were collected from 31 children $(4.4 \pm 0.8 \mathrm{yrs})$ during one hour of free-play. Video data were coded using the CARS. Cut points by Pate et al., van Cauwenberghe et al., Sirard et al. and Puyau et al. were applied to calculate time spent in sedentary, light and MVPA. Repeated-measures ANOVA and paired $t$ tests tested differences between the cut points and the CARS. Bland and Altman plots tested agreement between the cut points and the CARS. No significant difference was found between the CARS and the Puyau et al. cut points for sedentary, light and MVPA or between the CARS and the Sirard et al. cut point for MVPA. The present study suggests that the Sirard et al. and Puyau et al. cut points provide accurate group-level estimates of MVPA in preschool children.
\end{abstract}

Accurate means of quantifying physical activity levels in preschool children are important in research which seeks to explore the relationship between physical activity and health in the early years and whether levels of physical activity are adequate. Accelerometry offers one means of quantifying activity levels although methodological questions remain regarding how data are processed and interpreted for preschool children (8). In particular the determination of the number of minutes spent at different intensities is dependent on the thresholds applied to the accelerometry "activity counts" to convert these to a biologically meaningful outcome. Numerous cut points for Actigraph accelerometers have been developed for children and some for preschool children and there are a variety of cut points available, for example the cut points for moderate-to-vigorous activity (MVPA)

Hislop, Bulley, and Mercer are with the School of Health Sciences, Queen Margaret University, Edinburgh, Scotland, UK. Reilly is with the Physical Activity for Health Group, School of Psychological Sciences and Health, University of Strathclyde, Glasgow, Scotland, UK. 
range from activity above 1263 counts per minute (cpm; 13) to activity above 3600 cpm (18). Different cut points for time spent in sedentary behavior have also been reported for young children and these range from $<100 \mathrm{cpm}(10)$ to $<1592 \mathrm{cpm}$ (31). The application of different cut points makes comparison between studies problematic, leading to conflicting conclusions about levels of sedentary behavior, light activity and moderate-to-vigorous activity (MVPA; 14).

Discrepancies in cut points may in part be due to the differing criterion methods used during calibration studies such as direct observation (31) and indirect calorimetry (24) while children have engaged in treadmill-based or free-living activities, or both. Use of structured treadmill activities in calibration may not reflect the spontaneous 'free-living' activities typical of young children. Together with the use of a one-minute sampling period for data collection, this may reduce the accuracy of some of the proposed cut points (2). Many calibration studies have used linear regression equations to develop cut points, which may also be problematic, as the output from Actigraph accelerometers at higher intensities does not increase linearly (5). The application of equations developed for adults is not appropriate for children, and the use of three METs as a threshold for MVPA activity in young children may result in an overestimation of time spent in MVPA, as resting MET values are higher in childhood (14). To further complicate matters there is a lack of agreement on definitions of sedentary behavior, light activity and MVPA in preschool children. Sedentary behavior is either defined as predominantly sitting (25), or to include both standing and sitting activities (17); while MVPA can include slow walking (24) and in other studies slow walking is defined as a 'light' intensity activity (31). Finally some researchers argue that age-specific cut points are required (31), while others suggest that these are not needed (10); this issue remains unresolved.

A recent study has compared cut points for youth (33) and studies by Cliff and Okely (7) and Guinhouya et al. (15) have highlighted the discrepancies in quantification of MVPA when different cut points are applied. However, the crucial question of which cut point is most accurate, compared against an external criterion method, has yet to be discerned for preschool children. Direct observation is recognized as a criterion method for measuring physical activity which is particularly suited to young children (12). The Children's Activity Rating Scale (CARS) is a direct observation scale which has been validated for use in young children (27). While the CARS method is time-consuming and resource intensive and therefore not suitable for large population based studies, it has been widely used in methodological studies of young children $(21,22,31,34)$.

The present study therefore aimed to determine the accuracy of measurement of time spent in sedentary behavior, light intensity physical activity and MVPA upon the application of cut point thresholds developed specifically for preschool children by Pate et al. (24), van Cauwenberghe et al. (34), Sirard et al. (31), and Reilly et al. (29). The cut points by Puyau et al. (27), which are based on older children but which have been used in studies of preschool children were also are applied (11). Finally the cut point of $<100 \mathrm{cpm}$ developed from calibration studies which have used energy expenditure estimates (1.0-1.5METs) for sedentary behavior was also examined $(23,32)$. The sedentary cut point of $<100 \mathrm{cpm}$ is frequently cited as being appropriate for sedentary behavior $(10,23,32)$, and has been calibrated in younger children (5-9 years) by Evenson et al. (10). Comparison with the direct 
observation criterion method, using the Children's Activity Rating Scale (CARS; 26) was used to determine accuracy.

\section{Methods}

A convenience sample of 31 children aged three to five years was recruited from two preschools in Edinburgh, Scotland (15 boys, 16 girls, mean age $4.4 \pm 0.8$ yrs, height $104.8 \pm 6.3 \mathrm{~cm}$, weight $17.7 \pm 2.5 \mathrm{~kg}$, BMI $16.1 \pm 1.1 \mathrm{~kg} / \mathrm{m}^{2}$, with mean BMI z-score $0.20,90 \%$ being healthy weight, $10 \%$ classified as overweight/obese i.e., BMI at or above 85 th centile relative to UK population reference data). Children were included if they were apparently healthy, between the ages of three and five years, attending Edinburgh City Council preschools, and if parental written informed consent and the child's verbal assent to participate had been obtained. Children with any known physical problems which would affect their mobility were excluded from the study including neurological, respiratory and musculoskeletal problems. Procedures were in accordance with the ethical standards of the host institution's Research Ethics Committee from which ethical approval was granted.

Children were video recorded during one hour of unstructured free-play within their preschool setting while wearing a GT1M Accelerometer (ActiGraph, LLC, Walton Beach, Florida) on an elasticated belt around their waist. Video recording was undertaken during the preschool's time-tabled daily outdoor 'play-time'. Accelerometers were positioned in the midaxillary line at the level of the iliac crest. The GT1M (Actigraph, LLC, Walton Beach, Florida) is a small $(3.8 \times 3.7 \times 1.8 \mathrm{~cm})$, lightweight $(27 \mathrm{~g})$ uniaxial accelerometer which measures acceleration in the vertical plane (1). ActiGraph accelerometers have been validated and used extensively in studies with preschool children $(16,24)$. The raw data from the accelerometer is filtered and digitalised and converted to "activity counts" over a predefined period (epoch). Data were collected in one second epochs.

Using the video data, the children's activity was 'scored' for each 15-s period by a single researcher using the direct observation scale, the Children's Activity Rating Scale (CARS; 26). The CARS is considered to be a criterion measure of physical activity in preschool children (31), Puhl et al. (26) developed and validated the CARS against indirect calorimetry and heart rate, creating a five-point scale for use with young children. The CARS is presented in Table 1.

\section{Table 1 Children's Activity Rating Scale (CARS) 5-point Scale to Categorize Intensity of Physical Activity}

\begin{tabular}{ll}
\hline Level 1: & Stationary/motionless \\
Level 2: & Stationary/movement of limbs or trunk (very easy) \\
Level 3: & Translocation (slow/easy) \\
Level 4: & Translocation (medium speed/moderate) \\
Level 5: & Translocation (fast or very fast/hard) \\
\hline
\end{tabular}

Puyau et al. (27) 
Similar to the coding used in Sirard et al. (31) activity coded as being 1 (stationary/no movement) and 2 (stationary/movement limbs/trunk) on the CARS was classed as being 'sedentary'; activity coded as 3 (translocation/easy) was classified as being 'light' intensity activities. Activity coded as levels 4-5 represented MVPA, because in the original study by Puhl et al. (26) the mean energy cost of movement at CARS levels 4 and 5 was equivalent to at least three times the individual's resting energy expenditure. Using the adapted method for CARS with a 15-s epoch, described by Sirard et al. (31), children were scored for each level of activity lasting longer than three seconds within a 15-s period. Each level was scored once during the period and these scores were then averaged over the 15-s period to give a final score. Coding was undertaken for each 15 -s interval during the data collection period.

Data from the accelerometers were transferred to an Excel spreadsheet and the one-second epochs reintegrated into 15-s epochs, for comparison with the CARS data (9). Using a program developed with Visual Basic for Applications (VBA) the data were processed within Excel using the cut points for sedentary, light and MVPA intensity levels as defined by Puyau et al. (27)(Sed ${ }^{\mathrm{pu}}$, Light $\left.{ }^{\mathrm{pu}}, \mathrm{MVPA}^{\mathrm{pu}}\right)$, van Cauwenberghe et al. (34)(Sedva, Light ${ }^{\mathrm{va}}$, MVPA $\left.{ }^{\mathrm{va}}\right)$ and by Sirard et al. (31)(Seds, Lights $^{s}$, MVPA $^{\mathrm{s}}$ ) were applied (Table 2). In addition, the cut points for MVPA by Pate et al. (24)(MVPA ${ }^{\mathrm{pa}}$ ) and for sedentary behavior by Reilly et al. (29)(Sed ${ }^{\mathrm{r}}$ ) were applied. The cut point of $<100 \mathrm{cpm}$ (Sed $\left.{ }^{\mathrm{ev}}\right)$ for sedentary behavior was also examined as this cut point is cited as being appropriate for sedentary behavior $(10,23,32)$, and has been calibrated in children (5-9 years; 10$)$. The cut points were divided to allow analysis of 15-s epochs as undertaken in earlier studies (20).

Data were imported into SPSS version 17 for analysis. The mean $(S D)$ number of minutes of sedentary behavior, light intensity activity and MVPA was calculated. Using the Freidman's Repeated Measures ANOVA the difference between the number of minutes of sedentary behavior, light intensity activity and MVPA calculated from each of the cut points and direct observation at 15-s epochs was explored with post-hoc analysis using the Wilcoxon paired $t$ test. To reduce type I error, a Bonferroni correction was applied; therefore the significance level was set at $p<.01$.

To assess the accuracy of the different accelerometry cut points as an absolute measurement of physical activity, comparison was made between the number of minutes of sedentary behavior, light intensity activity and MVPA estimated by the different cut points and their relationship with the criterion measure of direct observation, using the Bland and Altman approach (4).

\section{Results}

Table 3 presents a summary of the physical activity levels of the sample during the period of direct observation while at preschool. There was a significant difference in the mean time spent in sedentary, light and MVPA as estimated by the different cut points and the CARS $(p=.00)$. Post hoc analysis revealed that there was no significant difference for sedentary activity between Sedentary ${ }^{\mathrm{pu}}$ and CARS ( $p=$ $.8)$ and for light activity between the Light ${ }^{\mathrm{pu}}$ and CARS $(p=.06)$. For MVPA there was no significant difference between the MVPA ${ }^{\mathrm{pu}}$ and CARS $(p=.06)$; and MVPA ${ }^{\mathrm{s}}$ 


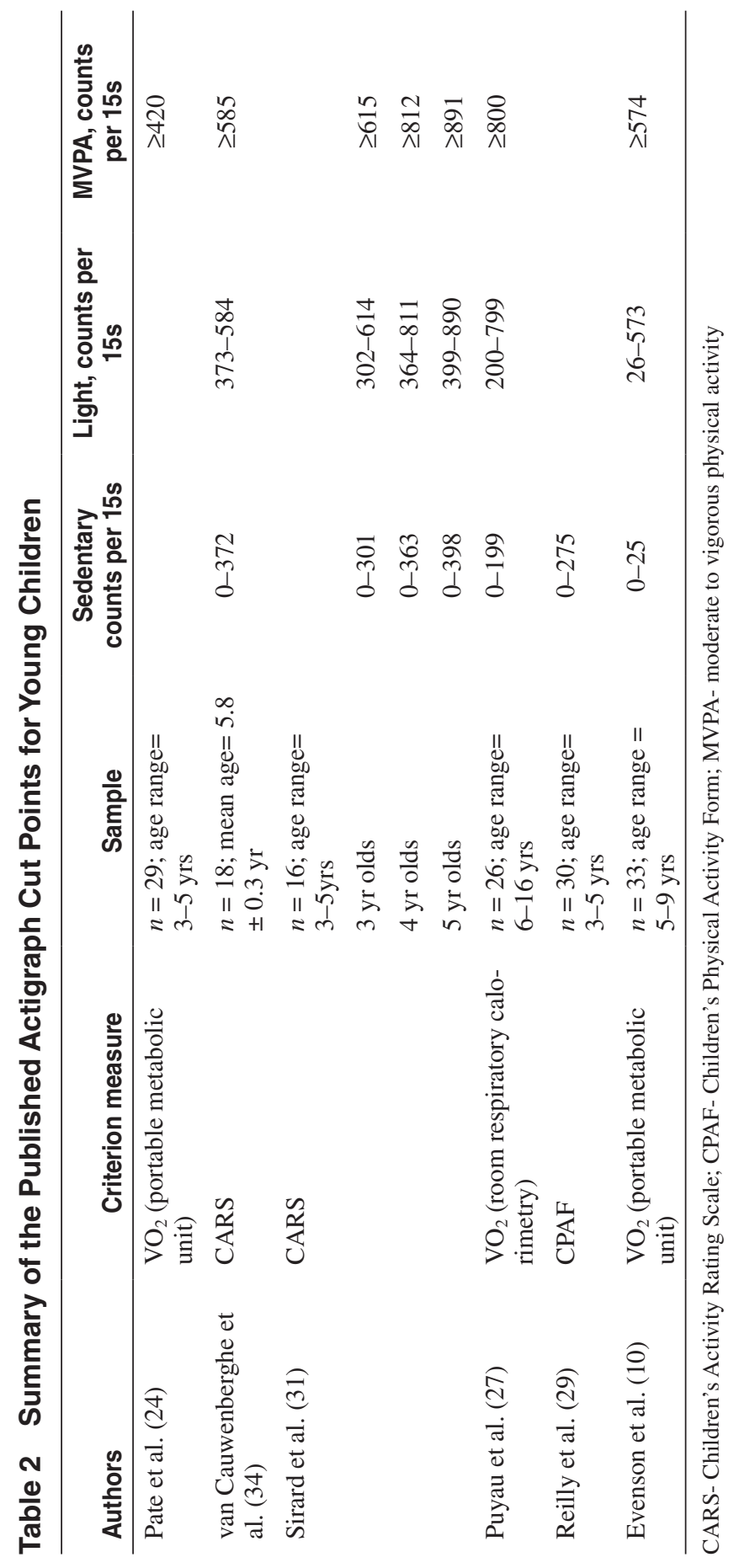


and the CARS $(p=.2)$. As the $\mathrm{p}$ value of 0.06 for the Light ${ }^{\mathrm{pu}}$ and MVPApu is close to 0.01 , it could suggest a trend in the data, despite there not being a significant difference from the CARS. There was a significant difference $(p<.01)$ between the CARS and the estimations made by the remaining cut points.

Bland and Altman plots were undertaken to examine the estimated number of minutes of sedentary behavior, light activity and MVPA produced by the different cut points against the CARS criterion measure. A summary of the Bland and Altman output is presented in Table 4. Plots are presented for Sirard et al. (31), Puyau et al. (27), van Cauwenberghe et al. (34), Reilly et al. (29), for Sedentary behavior (Figure 1a) and for Sirard et al. (31), Puyau et al. (27) and van Cauwenberghe et al. (34) for light and MVPA intensity activity (Figure 1b, and 1c).

While there was no obvious relationship between the difference and the mean for the cut points for sedentary behavior there was a bias toward a greater difference in estimation of time spent in sedentary behavior between the Sed ${ }^{\mathrm{s}}$, Sed ${ }^{\mathrm{va}}$ and Sed $^{\mathrm{r}}$ cut points and the CARS, and a lower mean difference in time in sedentary behavior for the Sed ${ }^{\text {ev }}$ and the CARS. The Sed ${ }^{\text {pu }}$ cut points were close to zero (mean difference $0.4 \mathrm{~min}$ ), however there were wide limits of agreement (LOA:-13.2-14.0 min). For light intensity activity all cut points had a lower estimation of time spent in light behavior in relation to the CARS. Finally, there was a positive bias, with an underestimation in MVPA between the MVPA ${ }^{\mathrm{s}}$ and MVPA ${ }^{\text {pu }}$ cut points and the CARS and a negative bias, overestimation in MVPA time for the MVPA ${ }^{\text {va }}$ and MVPApa $^{\text {pa }}$ cut points and the CARS.

\section{Discussion}

The results of the current study indicate relatively large errors at the individual level (wide limits of agreement) when accelerometry output was compared with the CARS. On a group level MVPApu and the MVPAs provided estimates which

Table 3 Mean Minutes of Sedentary Behavior, Light Intensity and Moderate to Vigorous Activity (MVPA) \pm SD for Cut Points

\begin{tabular}{lccc}
\hline & \multicolumn{3}{c}{ Time (mins) } \\
\cline { 2 - 4 } & Sedentary & Light & MVPA \\
\hline Puhl et al. (26)_CARS & $23.4(12.1)$ & $18.8(8.1)$ & $7.1(7.0)$ \\
Pate et al. (24) & - & - & $15.8(9.1)^{*}$ \\
van Cauwenberghe et al. (34) & $31.6(15.0)^{*}$ & $7.4(3.2)^{*}$ & $10.3(7.2)^{*}$ \\
Sirard et al. (31) & $30.6(14.6)^{*}$ & $12.4(4.8)^{*}$ & $6.3(6.2)$ \\
Puyau et al. (27) & $23.0(13.6)$ & $21.0(8.2)$ & $5.3(4.4)$ \\
Reilly et al. (29) & $27.0(14.3)^{*}$ & - & - \\
Evenson et al. (10) & $10.2(8.7)^{*}$ & - & - \\
\hline
\end{tabular}

CARS- Children's Activity Rating Scale.

*indicates those values which are significantly different from the CARS $\mathrm{p}<0.01$ Bonferroni correction. 
Table 4 Bland and Altman Output for the Mean Difference and 95\% LOA Between Cut Point Estimates and the CARS

\begin{tabular}{lcc}
\hline & \multicolumn{2}{c}{ Time (mins) } \\
\cline { 2 - 3 } & $\mathbf{d m}$ & $\mathbf{9 5 \%}$ LOA \\
\hline Sed & -7.2 & $-20.2-5.7$ \\
Sed $^{\text {pu }}$ versus CARS & 0.4 & $-13.25-13.99$ \\
Sed $^{\text {va }}$ versus CARS & -8.2 & $-22.7-6.2$ \\
Sed $^{\text {ev }}$ versus CARS & 13.2 & $-2.2-28.6$ \\
Sed $^{\mathrm{r}}$ versus CARS & -3.6 & $-17.6-10.4$ \\
Light $^{\text {v }}$ verus CARS & 6.3 & $-6.0-18.6$ \\
Light $^{\text {pu }}$ versus CARS & -2.2 & $-15.0-10.5$ \\
Light $^{\text {va }}$ versus CARS & 11.4 & -1.4 to24.2 \\
MVPAs $^{\mathrm{s}}$ versus CARS & 0.8 & $-6.2-7.8$ \\
MVPA $^{\text {pu }}$ versus CARS & 1.7 & $-8.0-11.5$ \\
MVPA $^{\text {va }}$ versus CARS & -3.3 & $-11.8-5.3$ \\
MVPA $^{\text {pa }}$ versus CARS & -8.7 & $-19.9-2.5$ \\
\hline
\end{tabular}

Footnote: $d m$ : mean difference; 95\% LOA: 95\% limits of agreement; CARS: Children's Activity Rating Scale, MVPA: moderate to vigorous activity. s: Sirard et al. (31) cut points; pu: Puyau et al. (27) cut points; va: van Cauwenberghe et al. (34) cut points, r: Reilly et al. (29) cut points, ev: Evenson et al. (10) cut points, pa: Pate et al. (24) cut points.

did not differ significantly compared with the criterion direct observation method, with a mean difference of $0.8 \mathrm{~min}$ for the Sirard et al. (31) cut points and $1.7 \mathrm{~min}$ for the Puyau et al. (27) cut points, bias suggesting an overestimation in time spent in MVPA from the CARS criterion measure. Only the Sed ${ }^{\text {pu }}$ and light ${ }^{\text {pu }}$ cut points provided accurate estimates of sedentary behavior and light intensity physical activities (mean difference of 0.4 and -2.2 min respectively). However with the $p=.06$ for light ${ }^{\mathrm{pu}}$ and $\mathrm{MVPA}^{\mathrm{pu}}$ being close to 0.01 could indicate a trend for a difference between the CARS and the estimated time in light intensity and MVPA using the Puyau et al. (27) cut points.

Although not presented in the results it is interesting to note that combining the light ${ }^{\mathrm{pu}}$ and MVPA ${ }^{\mathrm{pu}}$ resulted in 26.3 (11.3) minutes of mean 'total' activity and a nonsignificant difference $(p=.7)$ with the 25.8 (11.7) minutes CARS for mean 'total' activity. This is important given the recent recommendations for preschool children which have been expressed in terms of 'total' physical activity (combining light intensity and MVPA) (6).

Use of the MVPApa cut point produced a statistically significant difference and large biases relative to direct observation, with overestimation of time spent in MVPA. Application of the MVPApu, MVPA ${ }^{\text {pa }}$, MVPA va and MVPA s cut points to the different age groups in the sample; three year olds $(n=10)$, four year olds $(n=13)$, and five year olds $(n=8)$ revealed that the Sirard et al. (31) age-specific 


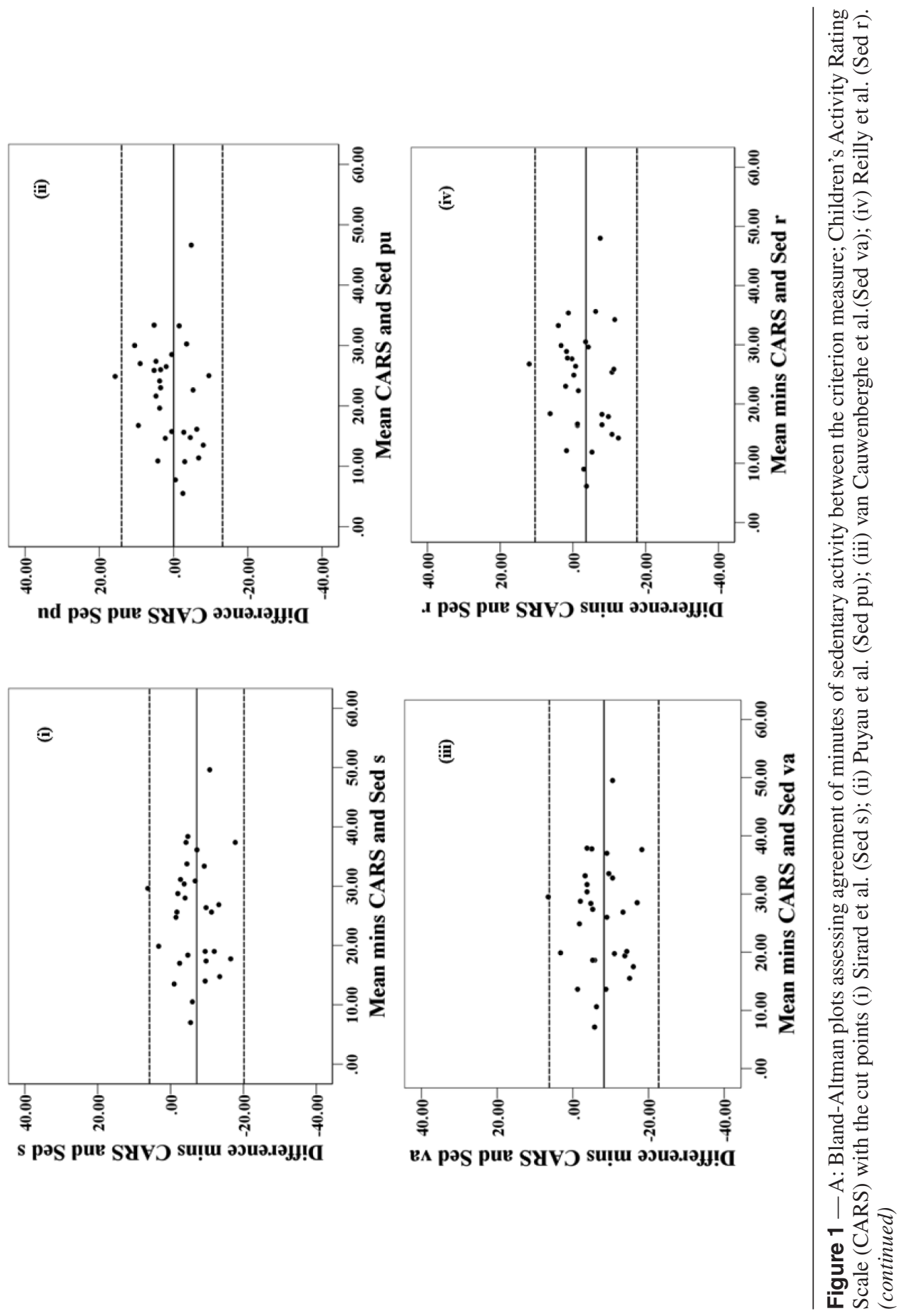




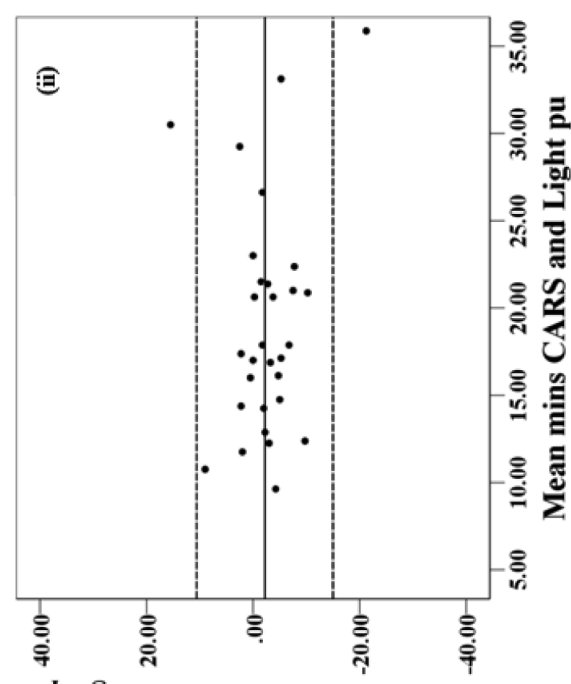

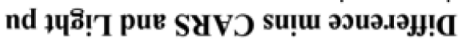
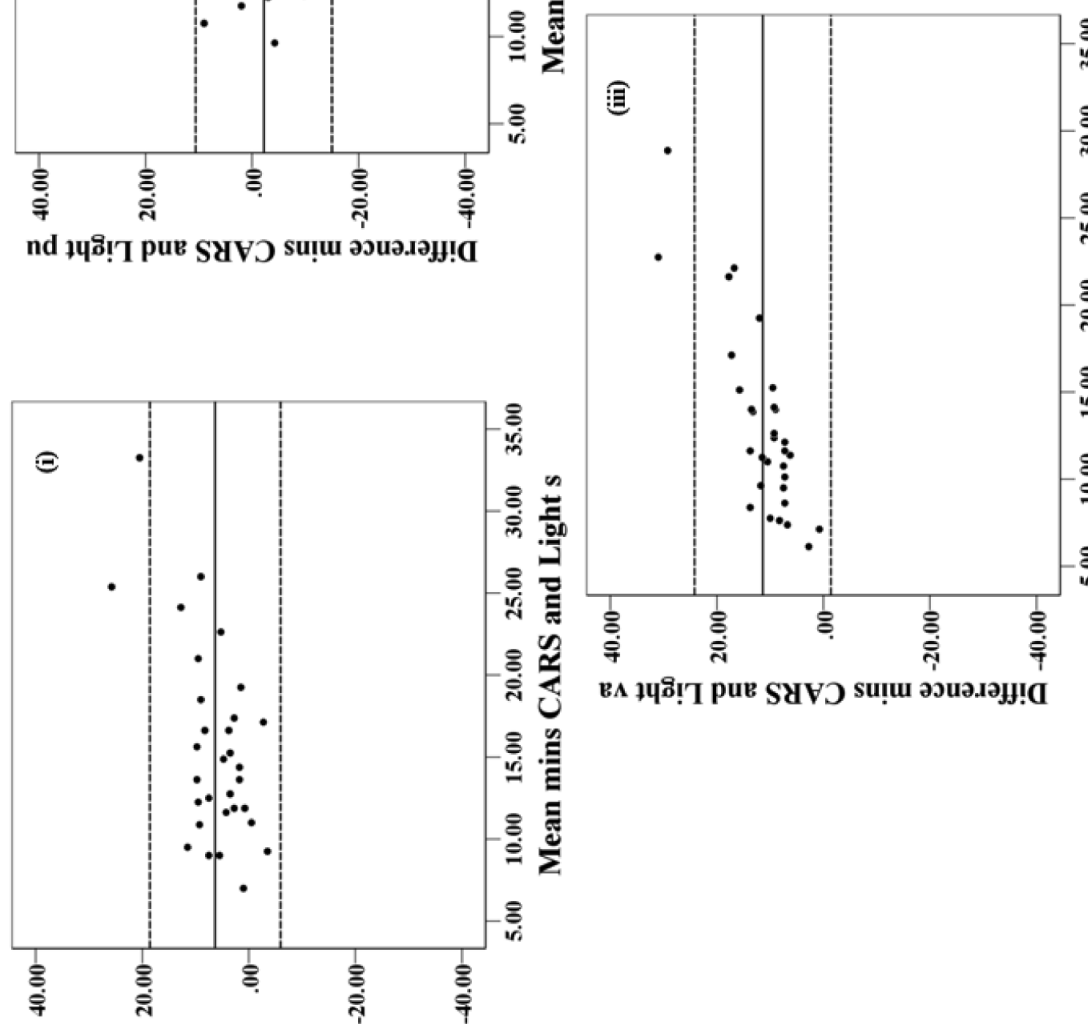

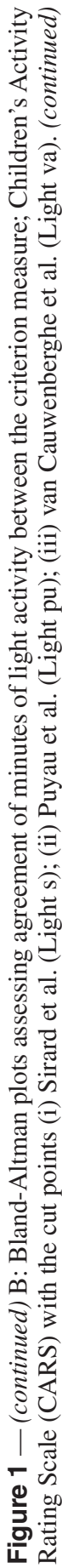




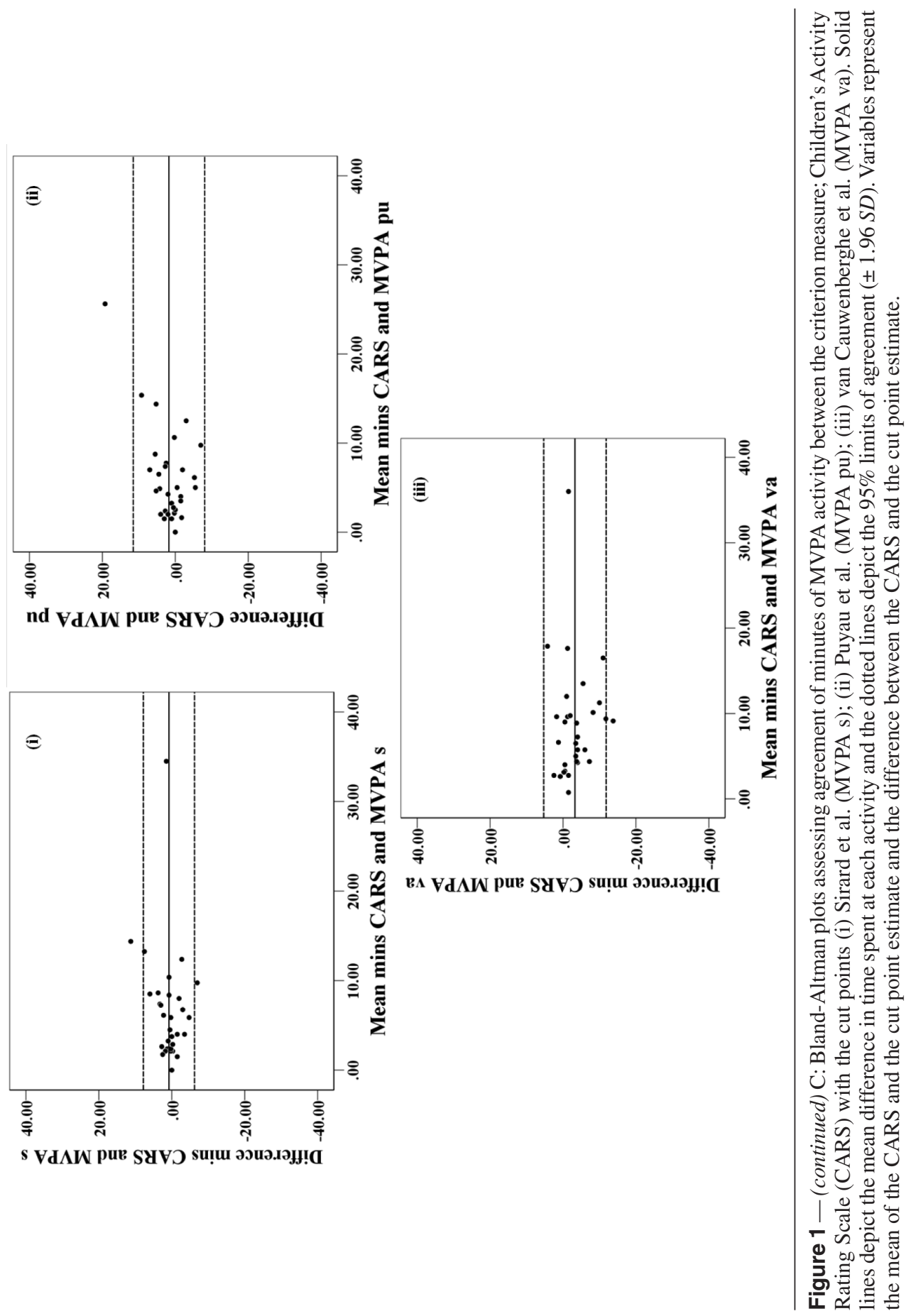


cut points for the three year olds in this study were the most accurate ( 0.7 min difference from the criterion) and the Puyau et al. (27) cut points were most accurate for five year olds ( 0.9 min difference from criterion). One of the outliers in the MVPA $^{\mathrm{pu}}$ Bland Altman plot, is a three year old for whom the higher Puyau et al. (27) cut points for MVPA resulted in a greater mean difference, i.e., an underestimation of time spent in MVPA in relation to the CARS, while the Sirard et al. (31) cut point led to a more accurate categorisation of intensity level for this child. It is however recognized that these subsamples are small and these results should be interpreted with caution.

Sirard et al. (31), Pate et al. (24) and van Cauwenberghe et al. (34) have identified cut point thresholds for Actigraph accelerometers specific to the preschool population. One explanation for the differences between estimates of physical activity derived from these cut points and CARS measures in the current study may be due to the differing calibration methods used in these studies to develop cut points. Pate et al. (24) used indirect calorimetry to calibrate their accelerometry cut points, which is a metabolic criterion measure. In contrast, Sirard et al. (31) and van Cauwenberghe et al. (34) used the CARS direct observation scale, a behavioral criterion measure. Freedson et al. (12) has suggested that behavioral approaches to calibration are particularly useful in studies of young children, where measurement and interpretation of energy expenditure can be difficult. Behavioral methods also avoid errors associated with extrapolation from treadmill activity to free-living behaviors. There are however limitations in direct observation methods, such as a risk of subject reactivity and problems with the accuracy of activity classification when the rating of intensity is subjective (35). The current study may be limited as it relied on the CARS direct observation scale as the 'criterion' measure where ideally this could have been combined with either $\mathrm{VO}_{2}$ or energy expenditure to gain a more comprehensive measure of physical activity. Interestingly, Puyau et al. (27) also used calirometry to calibrate accelerometry cut point, and the findings of the current study suggest that there was lack of significant bias between the number of minutes of MVPA with the Puyau et al. (27) cut points and the CARS criterion measure.

There are methodological differences between the current study and that of van Cauwenberghe et al. (34), which also used a modified CARS as the criterion method. In the current study MVPA was classified as activity at CARS level 4 and 5 as opposed to the classification of 'moderate' by van Cauwenberghe et al. (34) as averaged CARS scores of between 3.1-4.0 and vigorous 4.1-5.0. The lower CARS scores used by van Cauwenberghe et al. (34) for moderate intensity in their study meant that they included activities with an average energy expenditure of less than three times individual resting energy expenditure, which in the original CARS (26) was considered to be 'light' intensity physical activity. The current study used higher CARS thresholds to define MVPA since these represented an average energy expenditure of more than three times individual resting energy expenditure in the original CARS study, and so the approach taken to using CARS in the current study probably defines MVPA more adequately. In addition we used the validated approach outlined in Sirard et al. (31) which involved coding the observations over a 15-s period as opposed to a second-by-second direct observation coding used in the study by van Cauwenberghe et al. study (34).

Marked differences between cut points and the impact of these differences on apparent levels of MVPA and time spent in sedentary behavior have been highlighted 
by a number of earlier studies $(15,19,23,30)$ However these studies have simply described the differences between cut points, and in contrast to the current study, have not addressed the issue of the accuracy of the various cut points.

The time spent in sedentary behavior varied depending on which cut points were applied and these discrepancies are similar to findings reported in the review by Pate et al. (23). One of the limitations of the current study is that the CARS scale includes sitting and standing as sedentary activities, however sedentary behavior is argued to predominantly involve sitting (3) and it was not possible in this study to distinguish between these activities.

In the current study the mean minutes of MVPA $(7.0 \mathrm{~min})$ recorded during one hour of unstructured outdoor free-play time, where there is an opportunity for children to run and play, might seem relatively low, but if sustained over the whole day this could lead to accumulation of more than one hour of MVPA per day. Extrapolation of the findings to a $12 \mathrm{hr}$ day for preschool children would lead to estimates of time spent in MVPA ranging from $1.06 \mathrm{hr}$ with MVPAs, $1.26 \mathrm{hr}$ with MVPApu, $2.12 \mathrm{hr}$ with MVPA va and $3.16 \mathrm{hr}$ with MVPA $^{\text {pa }}$ over a day. This would result in a difference by a factor of 1.19 in estimated time spent in MVPA between MVPAs and MVPA ${ }^{\mathrm{pu}}$ cut points, which may not be biologically meaningful. However a difference by a factor of nearly 3 (2.98) in estimated time between the MVPA $^{\mathrm{s}}$ and MVPA ${ }^{\mathrm{pa}}$ cut points is of concern and could lead to drastically different conclusions with regard to time spent in MVPA. It is recognized that extrapolation of one hour of free play data will not accurately reflect total daily activity as it would be expected that MVPA might be relatively high during this activity. As a consequence it is likely that extrapolation would result in an overestimation of total daily MVPA and indeed the levels of MVPA in the current study are higher than those observed in most previous nursery-based studies (28). It is acknowledged that a limitation of the current study is that direct observation of one hour of free play limited the absolute amount of time in MVPA. While further work should examine longer periods of observation in larger samples, this study provides results which should inform the debate over which cut points to use to define physical activity and sedentary behavior in preschool children.

\section{Conclusion}

The present study indicates that the Pate et al. (24) and van Cauwenberghe et al. (34) cut points significantly overestimated minutes of MVPA in preschool children when compared against direct observation, a criterion measure of physical activity. Use of the Puyau et al. (27) and Sirard et al. (31) cut points produced estimates of MVPA which were not significantly different from the criterion measure at a group level. These findings have considerable implications when considering levels of MVPA among young children.

\section{Acknowledgments}

Funding for this study came from the Physiotherapy Research Foundation, charitable trust of the Chartered Society of Physiotherapy, United Kingdom. 


\section{References}

1. ActiGraph LLC. GT1M Technical Details [Internet]. 2007. [cited 2007 Mar 8]. Available from: www.theactigraph.com

2. Baquet, G., G. Stratton, P.E. Van, and S. Berthoin. Improving physical activity assessment in prepubertal children with high-frequency accelerometry monitoring: A methodological issue. Prev. Med. 44(2):143-147, 2007. PubMed doi:10.1016/j. ypmed.2006.10.004

3. Biddle, S.J., N. Pearson, G.M. Ross, and R. Braithwaite. Tracking of sedentary behaviours of young people: a systematic review. Prev. Med. 51(5):345-351, 2010. PubMed doi:10.1016/j.ypmed.2010.07.018

4. Bland, J.M., and D.G. Altman. Statistical Methods for Assessing Agreement Between Two Methods of Clinical Measurement. Lancet. XXX:307-310, 1986. PubMed doi:10.1016/S0140-6736(86)90837-8

5. Brage, S., N. Wedderkopp, P.W. Franks, L.B. Andersen, and K. Froberg. Reexamination of validity and reliability of the CSA monitor in walking and running. Med. Sci. Sports Exerc. 35(8):1447-1454, 2003. PubMed doi:10.1249/01.MSS.0000079078.62035.EC

6. Chief Medical Officers of England, Scotland, Wales and Ireland. Start Active, Stay Active. A report on physical activity for health from the four home countries' Chief Medical Officers. [Internet]. London: Department of Health; 2011. [cited 2012 Feb 2]. Available from: http://www.dh.gov.uk/en/Publicationsandstatistics/Publications/ PublicationsPolicyAndGuidance/DH_128209

7. Cliff, D.P., and A.D. Okely. Comparison of two sets of accelerometer cut-off points for calculating moderate-to-vigorous physical activity in young children. $J$ Phys Act Health. 4:509-513, 2007. PubMed

8. Cliff, D.P., J.J. Reilly, and A.D. Okely. Methodological considerations in using accelerometers to assess habitual physical activity in children aged 0-5 years. J. Sci. Med. Sport. 12(5):557-567, 2009. PubMed doi:10.1016/j.jsams.2008.10.008

9. Edwardson, C.L., and T. Gorely. Epoch Length and Its Effect on Physical Activity Intensity. Med. Sci. Sports Exerc. 42(5):928-934, 2010. PubMed doi:10.1249/ MSS.0b013e3181c301f5

10. Evenson, K.R., D.J. Catellier, K. Gill, K.S. Ondrak, and R.G. McMurray. Calibration of two objective measures of physical activity for children. J Sports Sci. 26(14):1557-1565, 2008. PubMed

11. Fisher, A., J.J. Reilly, C. Montgomery, et al. Seasonality in Physical Activity and Sedentary Behavior in Young Children. Pediatr. Exerc. Sci. 17:31-40, 2005.

12. Freedson, P., D. Pober, and K.F. Janz. Calibration of accelerometer output for children. Med. Sci. Sports Exerc. 37(Suppl. 11):S523-S530, 2005. PubMed doi:10.1249/01. mss.0000185658.28284.ba

13. Freedson, P.S., J. Sirard, E. Debold, et al. Calibration of the Computer Science and Applications, Inc. (CSA) accelerometer. Med. Sci. Sports Exerc. 29(Suppl.):S45, 1997.

14. Guinhouya, C.B., and H. Hubert. Incoherence with studies using actigraph mti among children age 6-12 yr. Med. Sci. Sports Exerc. 40(5):979, 2008. PubMed doi:10.1249/ MSS.0b013e318168da5a

15. Guinhouya, C.B., H. Hubert, S. Soubrier, C. Vilhelm, M. Lemdani, and A. Durocher. Moderate-to-vigorous physical activity among children: discrepancies in accelerometry-based cut-off points. Obesity (Silver Spring). 14(5):774-777, 2006. PubMed doi:10.1038/oby.2006.89

16. Jackson, D.M., J.J. Reilly, L.A. Kelly, C. Montgomery, S. Grant, and J.Y. Paton. Objectively measured physical activity in a representative sample of 3- to 4-year-old children. Obes. Res. 11(3):420-425, 2003. PubMed doi:10.1038/oby.2003.57 
17. Martin, A., M. McNeill, V. Penpraze, et al. Objective Measurement of Habitual Sedentary Behavior in Pre-School Children: Comparision of activPAL with Actigraph Monitors. Pediatr. Exerc. Sci. 23:468-476, 2011. PubMed

18. Mattocks, C., S. Leary, A. Ness, et al. Calibration of an accelerometer during freeliving activities in children. Int. J. Pediatr. Obes. 2(4):218-226, 2007. PubMed doi: $10.1080 / 17477160701408809$

19. Mota, J., M. Valente, L. Aires, P. Silva, M.P. Santos, and J.C. Ribeiro. Accelerometer cut-points and youth physical activity prevalence. Eur. Phys. Educ. Rev. 13(3):287-299, 2007. doi:10.1177/1356336X07081795

20. Nilsson, A., U. Ekelund, A. Yngve, and M. Sjöström. Assessing Physical Activity Among Children With Accelerometers Using Different Time Sampling Intervals and Placements. Pediatr. Exerc. Sci. 14(1):87-96, 2002.

21. O'Hara, N.M., T. Baranowski, B.G. Simons-Morton, B.S. Wilson, and G. Parcel. Validity of the observation of children's physical activity. Res. Q. Exerc. Sport. 60(1):42-47, 1989. PubMed

22. Oliver, M., J. Schluter, and G. Schofield. A New Approach for the Analysis of Accelerometery Data Measured on Preschool Children. J Phys Act Health. 8(2):296-304, 2011. PubMed

23. Pate, R.P., J.A. Mitchell, W. Byun, and M. Dowda. Sedentary behaviour in youth. Br. J. Sports Med. 45:906-913, 2011. PubMed doi:10.1136/bjsports-2011-090192

24. Pate, R.R., M.J. Almeida, K.L. McIver, K.A. Pfeiffer, and M. Dowda. Validation and Calibration of an Accelerometer in Preschool Children. Obesity (Silver Spring). 14(11):2000-2006, 2006. PubMed doi:10.1038/oby.2006.234

25. Pate, R.R., J.R. O'Neill, and J. Mitchell. Measurement of Physical Activity in Preschool Children. Med. Sci. Sports Exerc. 42(3):508-512, 2010. PubMed doi:10.1249/ MSS.0b013e3181cea116

26. Puhl, J., K. Greaves, M. Hoyt, and T. Baranowski. Children's Activity Rating Scale (CARS): description and calibration. Res. Q. Exerc. Sport. 61(1):26-36, 1990. PubMed

27. Puyau, M.R., A.L. Adolph, F.A. Vohra, and N.F. Butte. Validation and calibration of physical activity monitors in children. Obes. Res. 10(3):150-157, 2002. PubMed doi:10.1038/oby.2002.24

28. Reilly, J.J. Low Levels of Objectively Measured Physical Activity in Preschoolers in Child Care. Med. Sci. Sports Exerc. 42(3):502-507, 2010. PubMed doi:10.1249/ MSS.0b013e3181cea100

29. Reilly, J.J., J. Coyle, L. Kelly, G. Burke, S. Grant, and J.Y. Paton. An objective method for measurement of sedentary behavior in 3- to 4-year olds. Obes. Res. 11(10):1155-1158, 2003. PubMed doi:10.1038/oby.2003.158

30. Reilly, J.J., V. Penpraze, J. Hislop, G. Davies, S. Grant, and J.Y. Paton. Objective measurement of physical activity and sedentary behaviour: review with new data. Arch. Dis. Child. 93:614-619, 2008. PubMed doi:10.1136/adc.2007.133272

31. Sirard, J.R., S.G. Trost, K.A. Pfeiffer, M. Dowda, and R.R. Pate. Calibration and Evaluation of an Objective Measure of Physical Activity in Preschool Children. J Phys Act Health. 3:345-357, 2005.

32. Treuth, M.S., K. Schmitz, D.J. Catellier, et al. Defining accelerometer thresholds for activity intensities in adolescent girls. Med. Sci. Sports Exerc. 36(7):1259-1266, 2004. PubMed

33. Trost, S.G., P.D. Loprinzi, R. Moore, and K.A. Pfeiffer. Comparison of Accelerometer Cut Points for Predicting Activity Intensity in Youth. Med. Sci. Sports Exerc. 43(7):1360-1368, 2011. PubMed doi:10.1249/MSS.0b013e318206476e

34. van Cauwenberghe, E., V. Labarque, S.G. Trost, I. De Bourdeaudhuij, and G. Cardon. Calibration and comparison of accelerometer cut points in preschool children. Int. J. Pediatr. Obes. 6(2):e582-e589, 2010. PubMed doi:10.3109/17477166.2010.526223

35. Westerterp, K.R. Assessment of physical activity: a critical appraisal. Eur. J. Appl. Physiol. 105(6):823-828, 2009. PubMed doi:10.1007/s00421-009-1000-2 